\title{
In-orbit AIS performance of the Norwegian microsatellites NorSat-1 and NorSat-2
}

\author{
Torkild Eriksen $^{1}$ (1) Ø Øystein Helleren ${ }^{1} \cdot$ Andreas Nordmo Skauen $^{1} \cdot$ Frode A. S. Storesund $^{2} \cdot$ Anders Bjørnevik $^{2}$. \\ Harald Åsheim ${ }^{3}$. Eirik Voje Blindheim ${ }^{4}$. Jon Harr ${ }^{5}$
}

Received: 2 August 2019 / Revised: 7 November 2019 / Accepted: 10 November 2019 / Published online: 21 November 2019

(c) The Author(s) 2019

\begin{abstract}
Two Norwegian AIS-satellites, NorSat-1 and NorSat-2, were launched in July 2017. Both are equipped with the ASR x50, the latest space-AIS receiver developed by Kongsberg Seatex AS, offering advanced signal processing and continuous operation on all four AIS channels. The NorSat-satellites collect $\sim 1.5$ million messages from $\sim 50,000$ ships per day $(24 \mathrm{~h})$ each, which is a factor 2.8 increase in the number of messages compared to the ASR 100 on-board AISSat-1 and AISSat-2. The improvements of the AIS-satellites can be attributed to three developments: the performance of the receiver, the use of antenna diversity, and the use of frequency channel diversity. Daily statistics for February 2018 over the Mediterranean Sea illustrate the improvements: The median value of the number of messages received with NorSat- 1 using only one antenna is 2.3 times higher than for AISSat-1. When both NorSat-1 antennas are used, the improvement factor becomes 4.1 , and finally, when two additional receiver channels are used to collect long-range AIS messages, the total improvement becomes 8.2 times. In terms of ships detected, the factors are 1.8, 2.7, and 4.4 for the respective steps. Long-range AIS messages amount to just 5\% of the total AIS messages received by NorSat-1 in August 2017, but it allows to detect $20 \%$ more ships on a global scale, and as much as 10 times more ships in a the high-traffic area in the North Sea.
\end{abstract}

Keywords Maritime surveillance $\cdot$ Ship reporting systems $\cdot$ Satellite AIS $\cdot$ Performance evaluation

\section{Introduction}

The two Norwegian microsatellites NorSat-1 and NorSat-2 were launched 14 July 2017 [1], doubling the number of own assets for the satellite-based AIS data collection for the services provided by the Norwegian Coastal Administration. The satellite-AIS services were demonstrated with AISSat-1 in 2010 and continued through the addition of AISSat- 2 in

Torkild Eriksen

Torkild.Eriksen@ffi.no

Øystein Helleren

Oystein.Helleren@ffi.no

Andreas Nordmo Skauen

Andreas-Nordmo.Skauen@ffi.no

Frode A. S. Storesund

Frode.Storesund@kongsberg.com

Anders Bjørnevik

Anders.Bjornevik@km.kongsberg.com

Harald Åsheim

harald.aasheim@kystverket.no
2014. The NorSats carry the fourth-generation space-AIS receiver from Kongsberg Seatex AS, the ASR x50 developed under the ESA ARTES program using commercial off-theshelf components, efficient evaluation and testing schemes, and advanced technological solutions [2]. It is also known as the Novel SAT-AIS (NAIS) receiver [3] having high sensitivity, improved decoder performance, multi-antenna, and

Eirik Voje Blindheim

eirik.blindheim@statsat.no

Jon Harr

jon.harr@spaceagency.no

1 Norwegian Defence Research Establishment (FFI), P.O. Box 25, 2027 Kjeller, Norway

2 Kongsberg Seatex AS, Pirsenteret, 7462 Trondheim, Norway

3 Norwegian Coastal Administration, Flathauggata 12, 5523 Haugesund, Norway

4 Statsat AS, Drammensveien 165, 0277 Oslo, Norway

5 Norwegian Space Agency, Drammensveien 165, 0277 Oslo, Norway 
multi-channel support, built-in redundancy, $6 \mathrm{MHz}$ sampling mode, large mass memory, and long lifetime.

This paper investigate the improvements made globally as well as in selected areas in terms of the number of messages received and number of ships detected. It also quantifies the performance increase through three types of developments: the receiver electronics and the algorithms, the antenna diversity, and the multi-channel architecture giving continuous operation on all four AIS channels.

\section{Background}

Funded by the Norwegian Space Agency and the Norwegian Coastal Administration over the national budget, the prime goal of the NorSat-satellites is to enhance maritime surveillance in the High North through collection of AIS messages from ships in the area, but the satellites also provide global coverage. The data are used by the Norwegian Coastal Administration's Vessel Traffic Service (VTS) centres as well as by other national authorities.

The investment in AIS-satellites as a means of cost-effective public sector solutions has been highlighted in the parliamentary report that forms the basis for Norway's further focus on space activities [6] and the commitment to maintain the satellite-based AIS capacity is now in the National Transport Plan [7].

\section{Operational aspects}

The four satellites (AISSat-1, AISSat-2, NorSat-1, and NorSat-2) are operated from the Satellite Control Centre (SCC) at Statsat AS in Oslo. The ground and space segments are presented in Sects. 3.1 and 3.2, respectively.

\subsection{Ground segment}

The overall AIS-satellite mission architecture and main ground-site locations are shown in Fig. 1. The satellites receive AIS messages from ships at sea globally and forward the messages to the Vardø ground station $\left(70^{\circ} \mathrm{N}\right)$. All the received message data are downlinked. In principle, the Vardø location on average permits contact with one of the AIS-satellites in 12 of the satellites' 15 daily orbits. In practice, using the same ground station antennas for all S-band communications with all AIS-satellites, only one satellite can be contacted at a time in passes where more than one satellite is simultaneously within contact range. The AIS messages are forwarded to the SCC at Statsat AS in Oslo, and finally to the Norwegian Coastal Administration in Haugesund. Commands for tasking and operation of AISsatellites are sent in the opposite direction from the SCC.

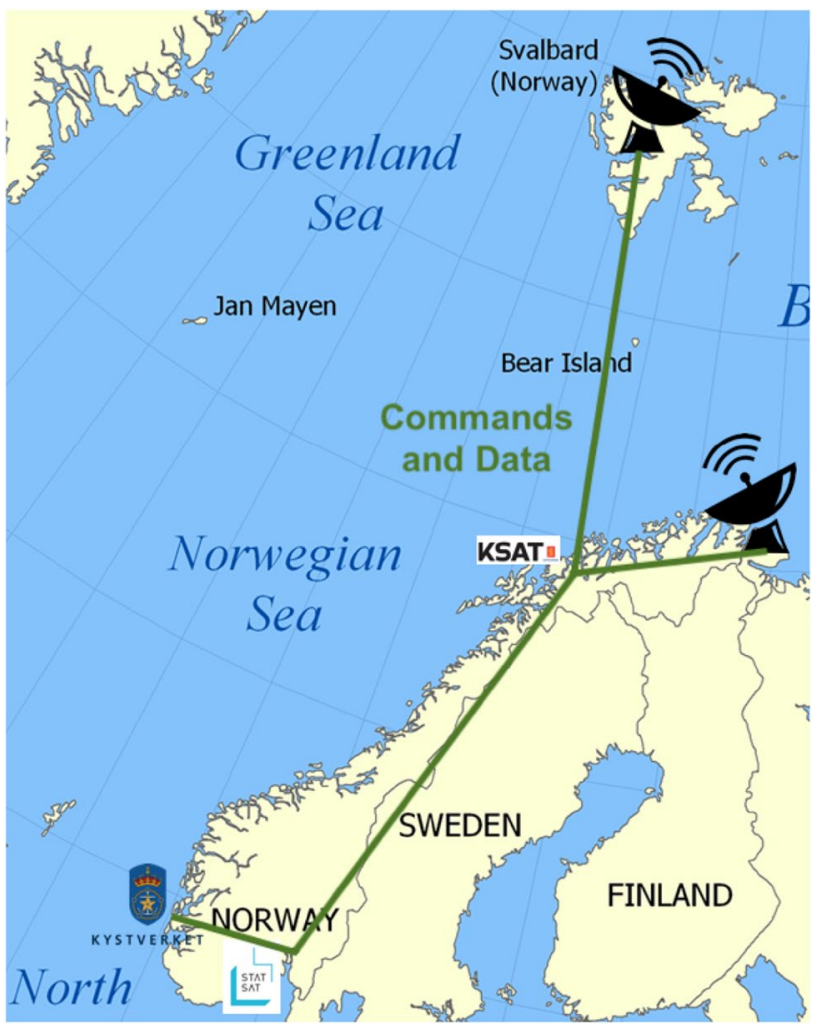

Fig. 1 Data flow diagram for AISSat-1 and -2, and NorSat-1 and -2

The Norwegian Coastal Administration is responsible for distributing both satellite- and terrestrial AIS data to users within the Coastal Administration as well as other Norwegian authorities and their partners.

\subsection{Space segment}

The satellites were custom built, tested, and prepared for flight by the University of Toronto Institute for Aerospace Studies/Space Flight Laboratory (UTIAS/SFL) on specifications from the Norwegian Defence Research Establishment (FFI) and the Norwegian Space Agency. While AISSat-1 and -2 are based on the $20 \mathrm{~cm}$ cube, mass $\sim 7 \mathrm{~kg}$, Generic Nano-satellite Bus (GNB), NorSat-1 and -2 are based on the more advanced and larger NEMO bus, which has dimensions $20 \times 25 \times 44 \mathrm{~cm}$ without the solar panels and a mass of $\sim 15 \mathrm{~kg}$. NorSat- 1 and -2 also have auxiliary payloads with operational attitude requirements. See Fig. 2 for illustrations of the satellites. The different AIS-antenna configurations and the operational attitude result in different AIS message reception performance between the NorSats and the AISSats, and between the individual NorSats.

UTIAS/SFL was also responsible for launch arrangements. AISSat-1 was launched by the Indian Polar Satellite Launch Vehicle (PSLV) from southern India July 2010, while AISSat- 2 entered orbit from a Soyuz launch vehicle 
Fig. 2 Illustration of NorSat-1 and -2 (courtesy: UTIAS/SFL)

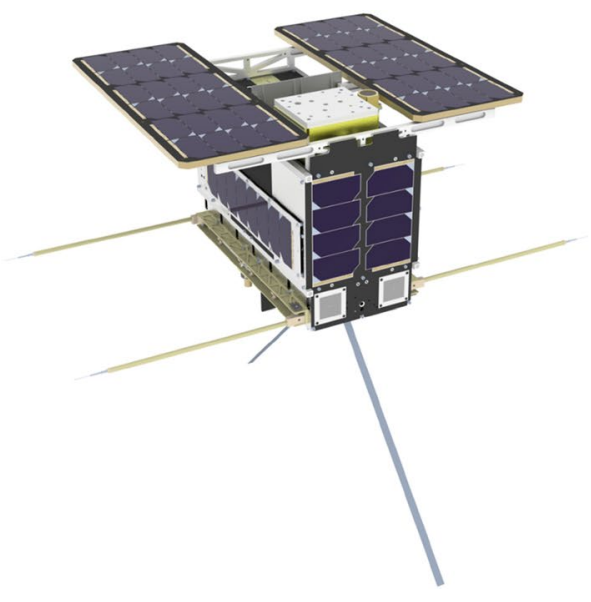

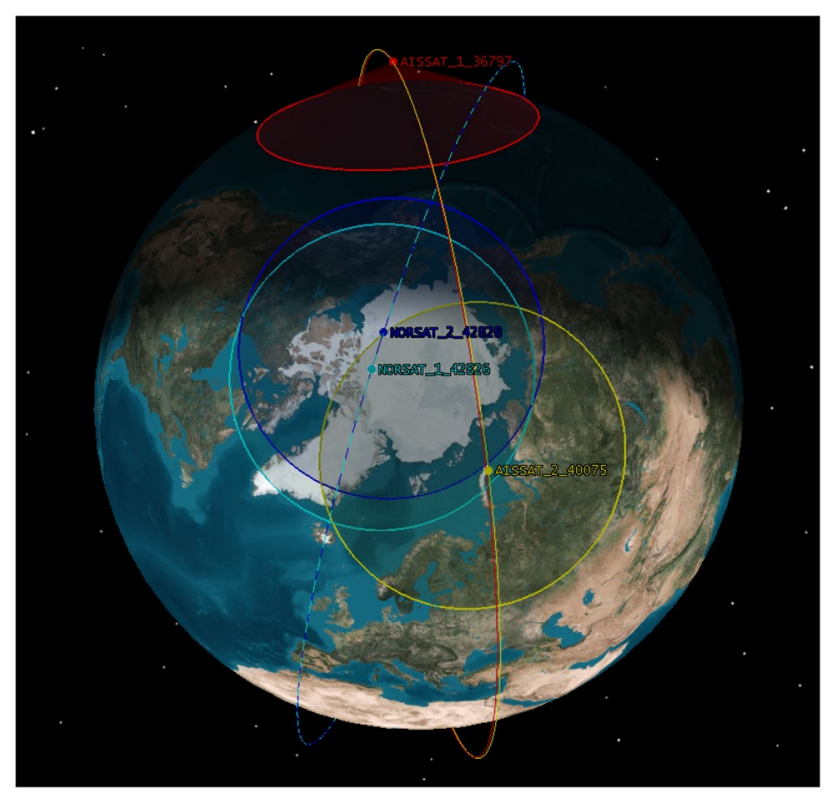

Fig. 3 Orbits and coverage for AISSat- 1 and -2 and NorSat- 1 and -2 on 22 July 2017 at 11:24 UTC

from Baikonur in Kazakhstan July 2014, and NorSat-1 and -2 utilized the Soyuz from Baikonur in July 2017. All launches were as secondary "piggyback" satellites.

\subsection{Satellite orbits}

The AIS receivers, the satellite orbits, and other parts of the system affect the message detection probability and the time between observations. The four satellites are all in polar sun-synchronous orbits with $98^{\circ}$ inclination, altitude of 590-630 km, and a revolution period of approximately 97 min. Figure 3 depicts the satellites' orbits and fields of view on 22 July 2017 at 11:24 UTC, shortly after the launch of the NorSats. The time has been chosen, so that all satellites are seen simultaneously.
At this time, AISSat-1 and -2 (red and yellow) are descending, following each other with a time separation of approximately $15 \mathrm{~min}$. NorSat- 1 and -2 (dark and light blue) are ascending, following each other with a time separation of approximately $1 \mathrm{~min}$. The AISSats and the NorSats, in pairs, have a fairly equal and repetitive coverage; about the same coverage area and the same local time of the equator crossing, but differences in altitude provide different revolution periods. The similar ground tracks, but varying coverage times, have these characteristics:

- AISSat-1 and -2 both had an ascending node at approximately 01:00 local time in summer 2017. Their difference in revolution period was $0.3 \mathrm{~min}$, resulting in a variation of the separation of the satellites with a period of 27 days, or $\sim 14$ days between overlapping field of view and $1 / 2$-orbit separation in the track.

- NorSat-1 and -2 had a rising node of 11:30 local time. Their difference in revolution periods was only $0.01 \mathrm{~min}$, which means that the distance between the satellites varies with a period of 432 days (based on the Two-LineElements (TLE) track data from 28 November 2017), giving 7 months between overlap and maximum separation.

\section{AIS messages}

The AIS standard ITU Recommendation ITU-R M.1371-5 [4], currently in version 5, approved February 2014, has 27 message types with different content and functions. AIS was originally intended for ship-to-ship and ship-to-shore data exchange; message type 27 "Position report for long-range applications" was added in version 4, approved April 2010, to increase the detection probability by satellites. In every AIS message, the ship is identified with the 9-digit Maritime Mobile Service Identity (MMSI) number that is unique for the AIS transponder on the ship. 
The standard has five message types that are ship position reports: types 1-3 and 27 are transmitted by Class A equipment, and type 18 by Class $B$ equipment. This is shown in Table 1. The nominal output power is $12.5 \mathrm{~W}$ for Class A and $2 \mathrm{~W}$ for Class $\mathrm{B}$ equipment. The lower power and longer reporting interval of the Class $\mathrm{B}$ equipment makes the ships more difficult to detect.

Class A equipment is used by ships that have carriage requirements (all commercial ships): Class B is a low-cost solution that is used voluntarily (by recreational boats). Both types of equipment transmit the original messages, type 1-26, alternatingly on two channels, referred to as AIS1 and AIS2. With the introduction of message type 27, two more channels, AIS3 and AIS4, were allocated exclusively for long-range AIS.

The global numbers of messages received during 1 day (24 h) shown in Table 3 include all types of position reports, see chapter 6 . The detailed performance studies in chapter 7 and 8 are for Class A position reports only.

AIS transponders compliant with version 3 of the standard, approved June 2007, or lower versions transmit only the original 26 message types on two channels. AISSat- 1 and -2 were designed to receive the messages from such equipment. Ships with AIS equipment in compliance with version 4 or higher transmit the long-range message. NorSat-1 and -2 , having multi-channel architecture, continuously receive these messages in addition to the original types.

\section{Satellite-AIS payloads}

The satellite-AIS payloads consist of AIS receivers developed and manufactured by Kongsberg Seatex AS and antennas from UTIAS/SFL.
AISSat- 1 and -2 carry the ASR 100 receiver, while NorSat- 1 and -2 are the first satellites using the ASR x50 receiver. The receivers are implemented as Software-Defined Radio (SDR) that supports, and has demonstrated, in-orbit updates of the algorithms to give upgraded performance. There are several differences between the ASR 100 and ASR x 50 receivers: number of channels, number of antenna inputs, and the digital signal processing for filtering including error correction of messages, see Table 2. Also, an ASR 300 receiver is installed on-board the International Space Station (ISS), from which data are presented together with data from the fours satellites in chapter 6 showing global numbers.

The AISSats have one linearly polarized monopole antenna. Both NorSats have two antennas: NorSat-1 uses two linearly polarized monopole antennas in an orthogonal configuration for antenna diversity. NorSat-2 has one linearly polarized monopole antenna and one high-gain Yagi antenna, the latter being part of the VDE-SAT payload [5], made by Kongsberg Seatex AS and owned and operated by Space Norway AS, which is the first test payload for the VHF Data Exchange System (VDES) satellite component.

\subsection{ASR 100 on-board AISSat-1 and AISSat-2}

The ASR 100 receiver [8] is the first-generation satelliteAIS receiver from Kongsberg Seatex AS, originally developed for AISSat-1, the first Norwegian AIS-satellites with operation as early as 2010. The specifications are shown in Table 2. The ASR 100 was also used on AISSat-2; it has been installed and operated as the NORAIS-1 Receiver onboard the ISS, and is launched as a commercial payload on other missions. The design was one of the first ever payloads to fully utilize all in-orbit reconfigurable software-defined technologies.

Table 1 AIS messages used in this work, as well as typical reporting intervals [4]

\begin{tabular}{|c|c|c|c|}
\hline Message type & Name & Description & Typical reporting intervals vs. speed \\
\hline 1 & Position report & Scheduled position report (Class A shipborne mobile equipment) & $\begin{array}{l}\text { At anchor: } 3 \mathrm{~min} \\
<14 \text { knots: } 10 \mathrm{~s} \\
<23 \text { knots: } 6 \mathrm{~s}\end{array}$ \\
\hline 2 & Position report & $\begin{array}{l}\text { Assigned scheduled position report (Class A shipborne mobile equip- } \\
\text { ment); data as in message } 1\end{array}$ & As above \\
\hline 3 & Position report & $\begin{array}{l}\text { Special position report, response to interrogation (Class A shipborne } \\
\text { mobile equipment); data as message } 1\end{array}$ & As above \\
\hline 18 & $\begin{array}{l}\text { Standard Class } \\
\text { B equipment } \\
\text { position } \\
\text { report }\end{array}$ & $\begin{array}{l}\text { Standard position report for Class B shipborne mobile equipment to be } \\
\text { used instead of Messages } 1,2,3\end{array}$ & $\begin{array}{l}<2 \text { knots: } 3 \mathrm{~min} \\
<14 \text { knots: } 30 \mathrm{~s} \\
<23 \text { knots: } 15 \mathrm{~s}\end{array}$ \\
\hline 27 & $\begin{array}{l}\text { Position } \\
\text { report for } \\
\text { long-range } \\
\text { applications }\end{array}$ & $\begin{array}{l}\text { Scheduled position report; (Class A shipborne mobile equipment outside } \\
\text { base station coverage) }\end{array}$ & $3 \mathrm{~min}$, independent on speed \\
\hline
\end{tabular}


Table 2 ASR 100 [8] and ASR $\mathrm{x} 50$ [9] technical specifications
Table 3 Number of messages and MMSIs detected globally with five satellites for 6 August 2017

\begin{tabular}{lll}
\hline Parameter & ASR 100 specification & ASR x50 specification \\
\hline Sensitivity & $-117 \mathrm{dBm} @ 20 \%$ PER & $<-126 \mathrm{dBm} @ 20 \%$ PER \\
Noise figure & $4.5 \mathrm{~dB}$ & $<2 \mathrm{~dB}$ \\
Eb/N0 & $12 \mathrm{~dB} @ 20 \%$ PER & $6 \mathrm{~dB} @<10 \%$ PER \\
& $8 \mathrm{~dB} @ 90 \%$ PER & $4 \mathrm{~dB} @<80 \%$ PER \\
Dynamic range & $-117 \mathrm{dBm}$ to $-90 \mathrm{dBm}$ & $-126 \mathrm{dBm}$ to $-40 \mathrm{dBm}$ \\
Interfaces & RS-485 & RS-422/RS-485, LVDS \\
Weight & $0.925 \mathrm{~kg}$ & $1.3 \mathrm{~kg}$ \\
Input voltage & $3.6-5.5 \mathrm{~V}$ DC & $9-32 \mathrm{VDC}$ \\
Power & $<1500 \mathrm{~mW}$ & $4.5-6.5 \mathrm{~W}$ \\
Failure rate & Not available & $<800 \mathrm{FIT} @ 40{ }^{\circ} \mathrm{C} \pm 5^{\circ} \mathrm{C}$ \\
RF antenna interface & Single antenna support & 4 antenna support \\
Parallel receivers & 2 & 4 \\
Optional bit correction & No & Yes \\
Sampling capacity & $20 \mathrm{~s}$ & 100 min \\
Design lifetime & 3 years in LEO & $7+$ years in LEO \\
\hline
\end{tabular}

\begin{tabular}{|c|c|c|c|c|c|c|c|c|}
\hline & \multicolumn{2}{|c|}{ Message type 1-3 } & \multicolumn{2}{|c|}{ Message type 27} & \multicolumn{2}{|c|}{ Message type 18} & \multicolumn{2}{|l|}{ Total } \\
\hline & Msg & MMSI & Msg & MMSI & Msg & MMSI & Msg & MMSI \\
\hline AISSat-1 & 382,080 & 24,607 & & & 10,049 & 3151 & 392,129 & 27,718 \\
\hline AISSat-2 & 514,276 & 25,076 & & & 14,511 & 3564 & 528,787 & 28,588 \\
\hline NORAIS-2 & 564,106 & 28,837 & 52,174 & 11,734 & 5405 & 2048 & 621,685 & 37,930 \\
\hline NorSat-1 & $1,304,814$ & 36,352 & 118,914 & 13,287 & 48,545 & 7263 & $1,472,273$ & 50,864 \\
\hline NorSat-2 & $1,309,345$ & 36,312 & 52,133 & 11,908 & 47,002 & 7027 & $1,408,480$ & 49,488 \\
\hline Total & $4,074,621$ & 43,895 & 223,221 & 13,521 & 125,512 & 9349 & $4,423,354$ & 59,505 \\
\hline
\end{tabular}

\subsection{ASR 300 on-board ISS}

In the hardware upgrade of the Norwegian AIS demonstrator on the ISS, the ASR 100, named NORAIS-1, was replaced by an ASR 300 referred to as NORAIS-2. This is a thirdgeneration receiver that, as a predecessor of ASR x50, has continuous operation on four channels, and has collected long-range messages continuously since February 2015.

\subsection{ASR x50 on-board NorSat-1 and NorSat-2}

The ASR x50 receiver [9] is the fourth-generation satelliteAIS receiver from Kongsberg Seatex AS and part of their new extended lifetime product series. It has a larger Field Programmable Gate Array (FPGA) than the previous generations and supports simultaneous on-board AIS decoding and digital sampling.

It achieves leading-edge performance through an improved RF front end with superior dynamic range, new enhanced algorithms, multi-antenna support, and multichannel architecture that enables simultaneous reception of the original messages on AIS1 and -2 and the long-range AIS messages on AIS3 and -4 . The receiver supports up to four antennas on each AIS channel, and has multiple advanced demodulator algorithms increasing the reception performance [10].

Like its predecessors, the receiver can be upgraded in orbit and thereby support new revisions of the AIS standard or other maritime VHF applications. The design lifetime is 7.5 years; technical specifications are shown in Table 2 .

\section{Global performance}

Shipping companies, traders, and authorities with responsibilities for maritime traffic as well as climate and environment have an interest in global ship tracking and ship densities. This chapter gives an example of the number of messages received and ships detected during 1 day, 6 August 2017 , shortly after the launch of NorSat-1 and -2 . The global overview comprises the data collected by the NORAIS-2 Receiver on-board the ISS as well as the four Norwegian satellites, which is addressed as five satellites in Table 3 and Fig. 4. The next chapters give more detailed examples of the performance of the four Norwegian satellites with emphasize on the improvements made. 


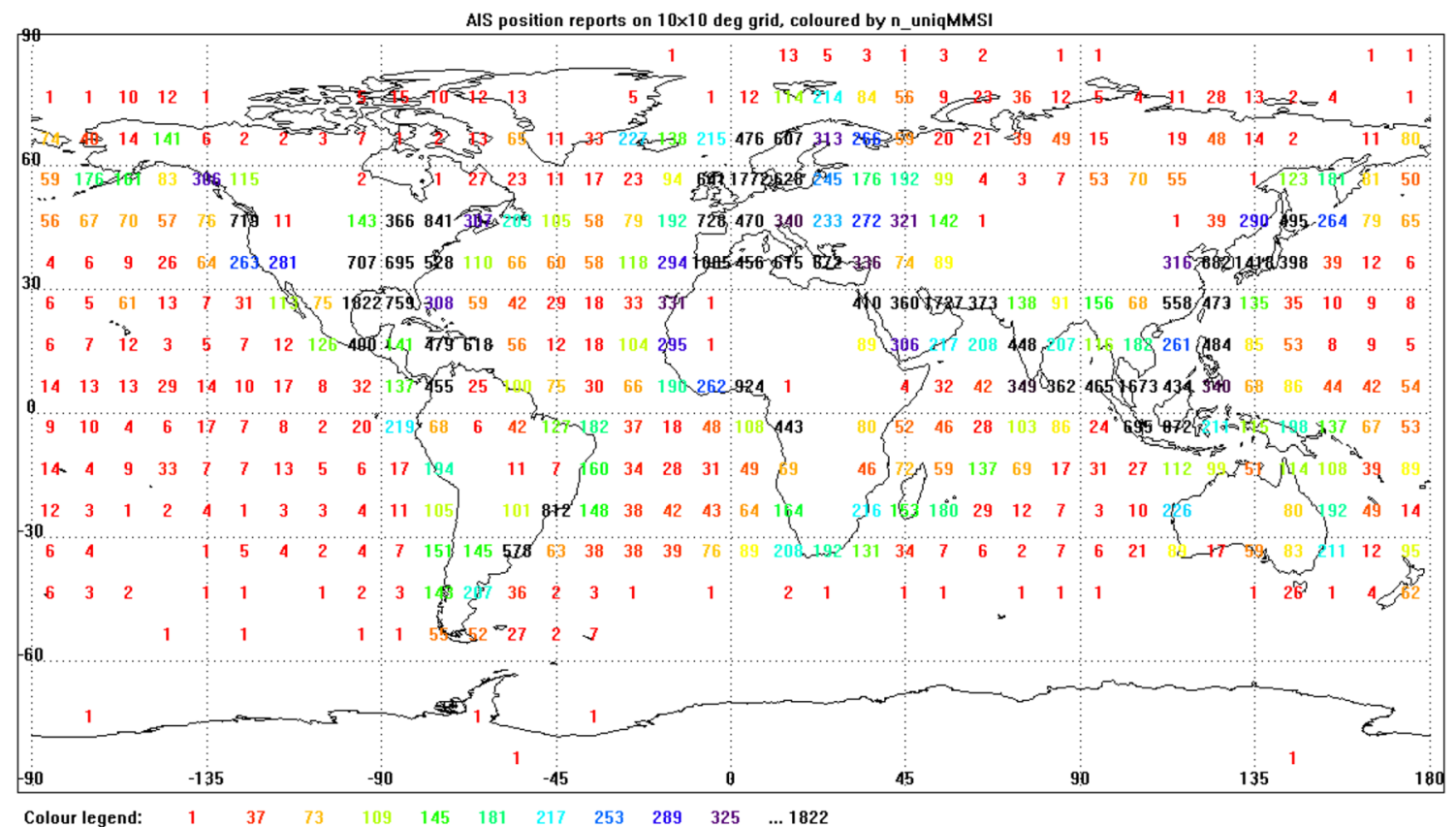

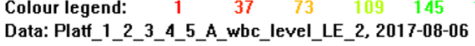

Fig. 4 Number of ships detected on $10^{\circ} \times 10^{\circ}$ grid on 6 August 2017 from position reports from five satellites

Table 3 shows the number of messages received and ships detected, grouped by the three types of position reports listed in Table 1. The receivers were operated the whole day, except AISSat-1 that had somewhat reduced operations in the southern hemisphere to save power due to aging batteries. Figure 4 shows the global distribution of the number of Class A equipped ships detected on $10^{\circ} \times 10^{\circ}$ grid.

More than 4 million messages of type 1-3, 125,512 of type 18, and 223,221 of type 27 are received. Messages type 1-3 are from 43,895 different ships (MMSI's), type 18 are from 9349 ships, and type 27 from 13,521 ships; the total number of ships is 59,505. As the Class B equipped (recreational) vessels amount to 9349, the number of Class A equipped (commercial) vessels amount to 50,156 (59,505-9349), of which $6261(50,156-43,895)$ are only detected on the long-range channels.

The trend for the global number of ships detected on the long-range channels shows a steadily growth: Using typical values for August as example, the number was $\sim 13,000$ ships/day in 2017, 18,000 ships/day 2018, and 23,000 ships/day in 2019 . For message types $1-3$, such a trend is not seen.

For all ships, class A and B together, NorSat- 1 with ASR $\mathrm{x} 50$ receiver collected $2.8(1,472,273 / 528,787)$ times as many messages and detected $78 \%((50,864-28,588) / 28,588)$ more ships than AISSat- 2 with ASR 100 receiver on 6 August 2017 (both satellites were operated the entire 24-h period).
The change in performance in some selected areas is shown in the next chapters, emphasizing how technology development as well as the long-range message type contribute to the improvement. Note that ships with Class B equipment are not used in the analysis of the improvements of the receivers in the subsequent chapters.

\section{Performance in selected areas}

Whereas stakeholders in shipping have a global perspective, national and regional authorities more often have responsibilities within defined areas, such as exclusive economic zones, NAVAREAs, fisheries zones and fisheries protection areas, areas with piracy, etc.

The detection probability of the AIS messages varies with the traffic density that causes co-channel interference, as well as terrestrial emissions causing noise in the AIS channels. The studies here are based on data from February 2018. Data from 10 February are used as example of a typical day.

A temporal resolution of 1 day is used as basis for the analysis of the performance of the satellites. In Figs. 5 and 6, the top panel shows data from AISSat-1, representing the first-generation space-AIS receiver, the middle panel shows data from NorSat-1, representing the fourth-generation receiver, and the bottom panel shows data for the service from the four satellites together. Note that AISSat-1 contributes as much as AISSat-2 in European waters; the lower 


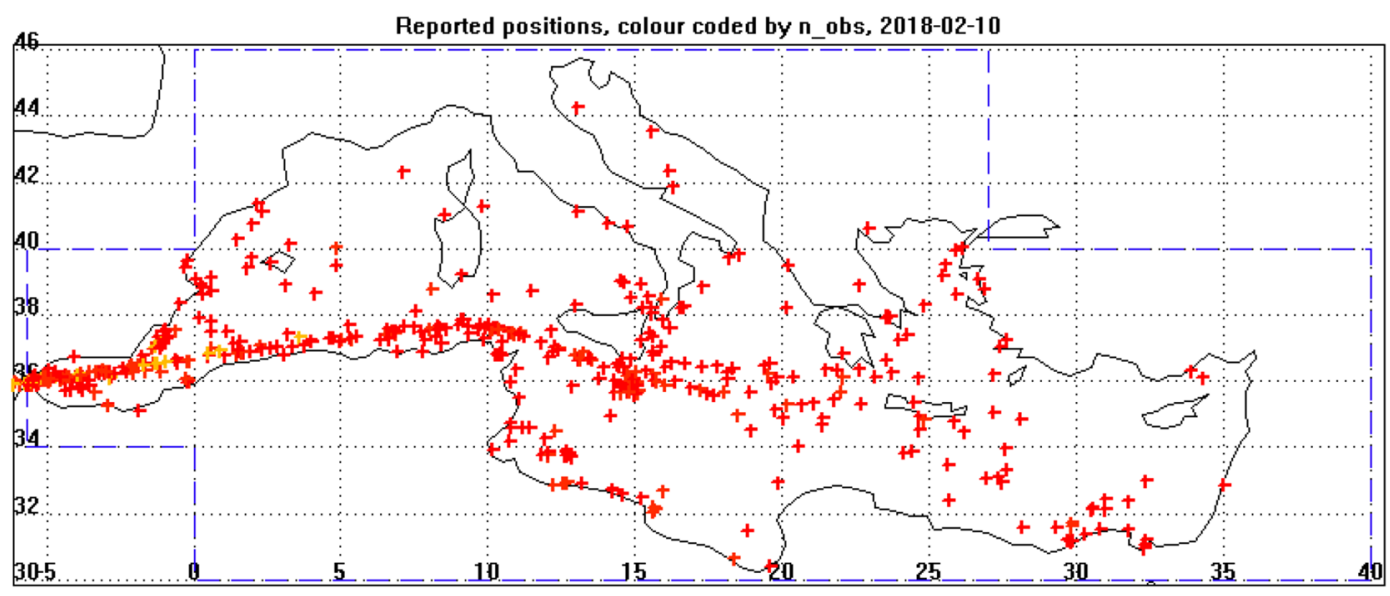

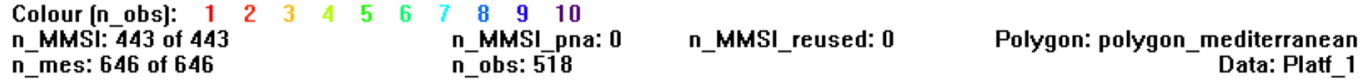

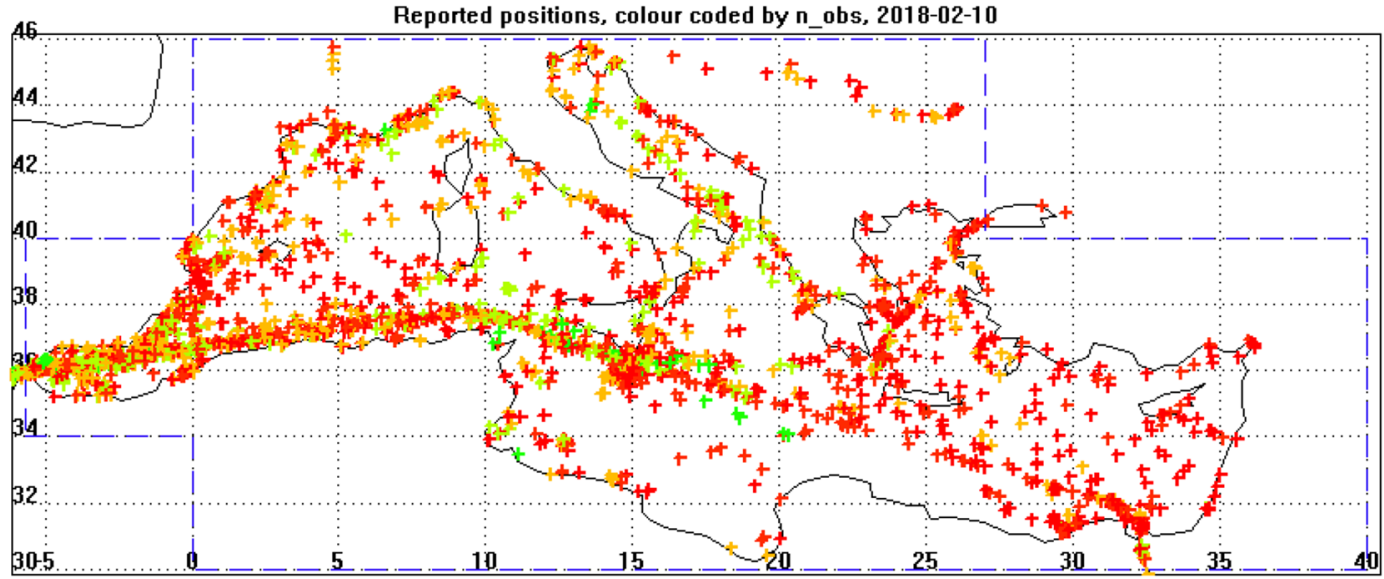

Colour [n_obs]: $\begin{array}{rrrrrrrrrrr}1 & 2 & 3 & 4 & 5 & 6 & 7 & \mathbf{8} & \mathbf{9} & \mathbf{1 0}\end{array}$

n_MMSI: 1558 of 1563 n_MMSI_pna: 2 n_MMSI_reused: 5 Polygon: polygon_mediterranean

n_mes: 5288 of 5913 n_obs: $3011 \quad$ Data: Platf_4_A_Two_Ant

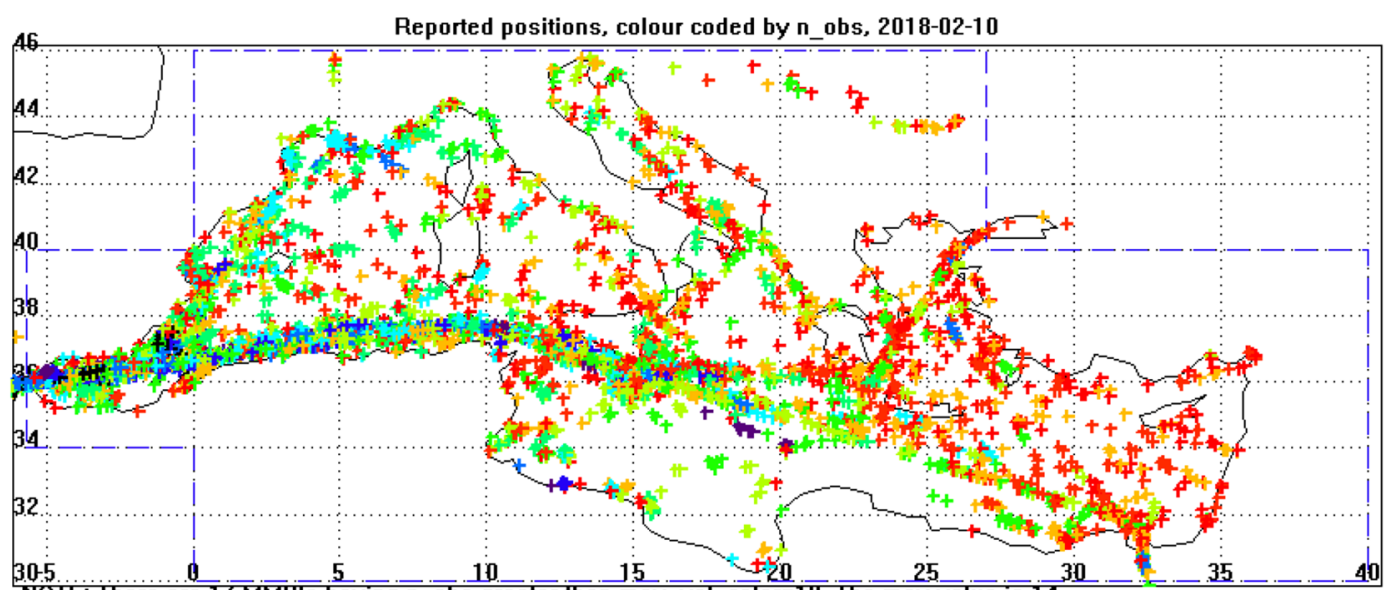

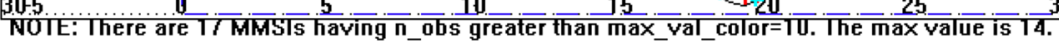

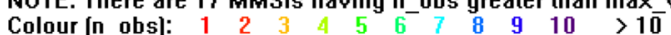

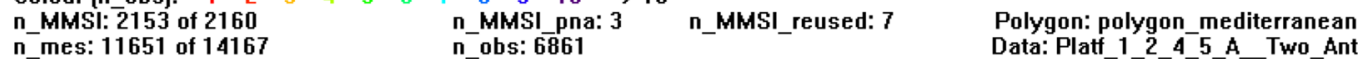

Fig. 5 Ship positions in the Mediterranean Sea on 10 February 2018 colored by the number of observations per ship. Top: AISSat-1; middle: NorSat-1; bottom: AISSat-1 and -2 and NorSat-1 and -2 (four satellites) together 
Fig. 6 Ship positions in the North Atlantic on 10 February 2018 colored by the number of observations per ship. Top: AISSat-1; middle: NorSat-1; bottom: AISSat- 1 and -2 and NorSat-1 and -2 (four satellites) together

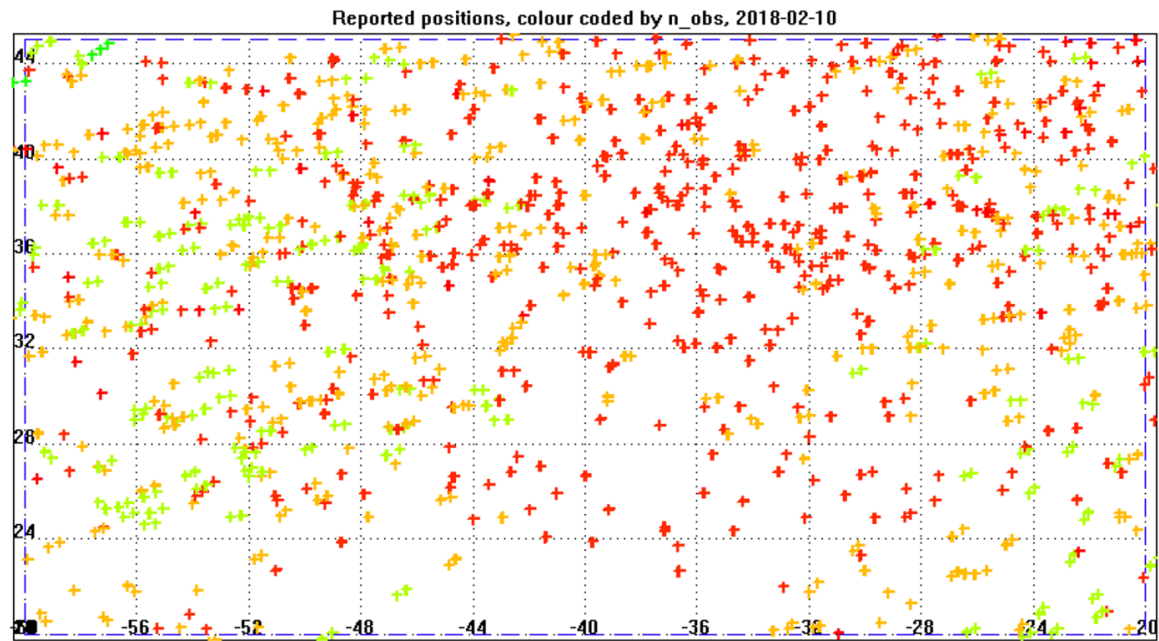

Colour (n_obs): $1 \begin{array}{cccccccccc}\mathbf{1} & 2 & 3 & 4 & 5 & 6 & 7 & \mathbf{8} & \mathbf{9} & \mathbf{1 0}\end{array}$

n_MMSI: 519 of 520 n_MMSI_pna: 0 n_MMSI_reusedybon: north_atlantic_020N_045N 60W 20W n_mes: 7681 of 7699 n_obs: 1265 Data: Platf_

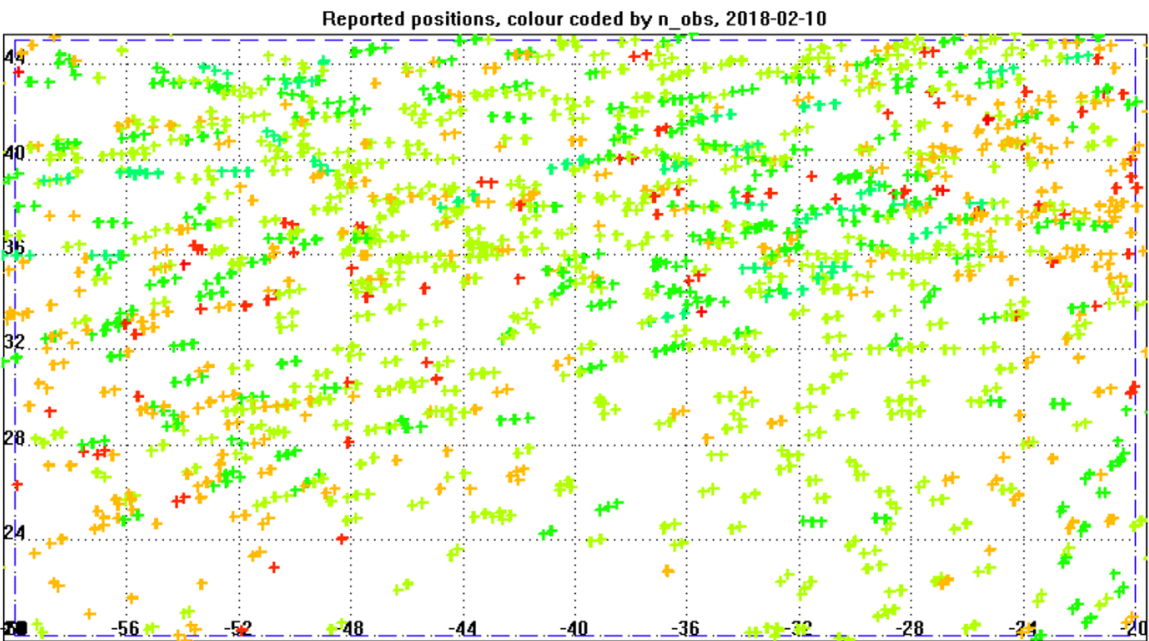

Colour [n_obs]: $\begin{array}{rlllllllllll}1 & 2 & 3 & 4 & 5 & 6 & 7 & \mathbf{8} & \mathbf{9} & \mathbf{1 0}\end{array}$

n_MMSI_pna: 2 n_MMSI_reuse_lygon: north_atlantic_020N_045N_60W_20W n_mes: 21487 of 2153

Reported positions, colour coded by n_obs, 2018-02-10

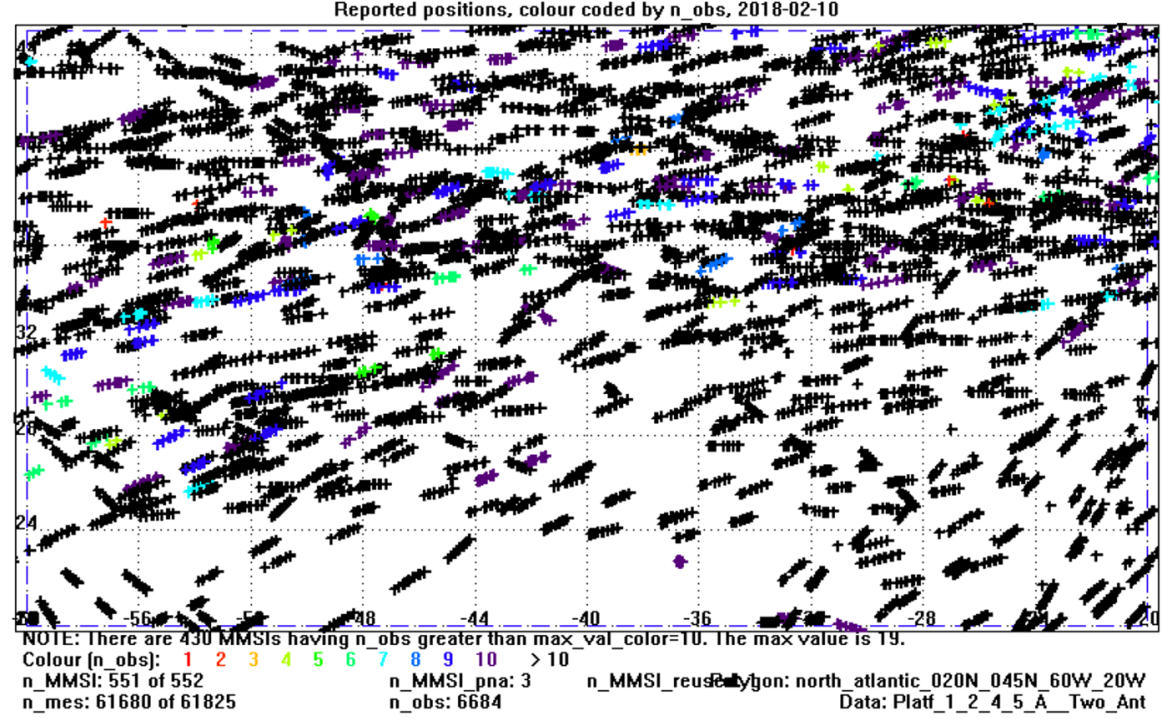


global numbers shown in chapter 6 are caused by somewhat reduced AISSat-1 operations in the southern hemisphere.

The Mediterranean Sea is used to illustrate the performance in a high-traffic area, whereas the central part of the North Atlantic illustrates the performance over the oceans. The satellites were collecting data during every access to the areas.

The distribution of the number of messages received per ship per day is not bell shaped (in statistics called normally distributed) but instead skewed, often with a long tail, and the central tendency is, therefore, better described by the median value than the mean [11].

Other methods for quantifying the tracking capability of satellite-AIS systems, defined as the probability of re-detecting ships as they move around the globe, are presented with examples from AISSat-1 and -2 in [12]; an update including NorSat-1 and -2 is made in [13].

\subsection{High-traffic area}

Ship positions for a typical day in the Mediterranean Sea are shown in Fig. 5. Table 4, chapter 8, shows that the median number of messages for NorSat-1 in the Mediterranean Sea in February 2018 is 8.2 times higher and the number of ships (i.e., unique MMSI numbers) detected is 4.4 times higher than for AISSat-1. The improvements are seen when comparing the two upper panels of Fig. 5; the number of MMSIs (n_MMSI) and the total number of messages (n_mes), and observations (n_obs) are shown below each plot. The polygon used to extract the MMSIs is shown as a blue dashed line, but messages received from the ships during the whole day are used for the calculations, as can be seen outside the polygon near Istanbul, Turkey.

A rainbow color scale representing the number of observations for each ship is used for the plotted ship positions. The detection probability is increased in the entire Mediterranean Sea, but in particular in the north as can be seen in the Adriatic Sea as well as on the Donau River. The colors show how the detection probability varies, with higher number of observations in the western than in the eastern parts.
The improvements are also seen in the number of ships for which more than one observation is made per day. The median values show that the number of ships observed by AISSat-1 was 362 per day, of which 301 (83\%) with only one observation per day. For NorSat-1, the numbers are 1580 ships, of which 911 (58\%) with one observation. For all four satellites together, the numbers are 2203 in total, of which 801 (36\%) with one observation. Hence, 2200 ships are tracked on a daily basis, and $\sim 1400$ of these are updated during the day.

Collection of message 27 increases the number of detected ships, in particular in high-traffic areas. In the Mediterranean Sea, the median number of ships per day increases from 983 to 1580 , an increase of $61 \%$, as shown in chapter 8 . In the North Sea, corresponding numbers are 100 ships without and 978.5 with the long-range messages considered [15].

\subsection{Ocean area}

In the central part of the North Atlantic, the median number of messages received per day is 3.1 times higher for NorSat-1 than for AISSat-1, as shown in Table 4, chapter 8, and illustrated in Fig. 6. The number of ships detected is, however, only increased by $6 \%$. This is because AISSat-1 performs well enough to detect most ships on a daily basis, which is the timeframe of the quantisation. The improvements are seen comparing the two upper panels of Fig. 5.

The median values for February 2018 show that the number of ships observed by AISSat- 1 was 471 ships per day, of which $49(10 \%)$ with only one observation. For NorSat-1, the median values are 497.5 ships per day, of which $13.5(3 \%)$ had only one observation. For all four satellites together, the numbers are 517 ships per day, of which 3 $(0.5 \%)$ with one observation. Hence, $\sim 517$ ships are tracked on a daily basis, and $\sim 514$ of these are updated during the day. This is thanks to a benign signal environment as well as good receiver performance.
Table 4 Comparison of number of messages received and ships detected (Count) and the improvement factor of NorSat-1 in different configurations relative to AISSat-1 (Factor) Median values for February 2018

\begin{tabular}{|c|c|c|c|c|c|c|c|c|}
\hline \multirow{3}{*}{$\begin{array}{l}\text { Area and statistics satellite } \\
\text { and configuration }\end{array}$} & \multicolumn{4}{|c|}{ Mediterranean Sea } & \multicolumn{4}{|c|}{ North Atlantic } \\
\hline & \multicolumn{2}{|c|}{ Messages } & \multicolumn{2}{|l|}{ Ships } & \multicolumn{2}{|c|}{ Messages } & \multicolumn{2}{|l|}{ Ships } \\
\hline & Count & Factor & Count & Factor & Count & Factor & Count & Factor \\
\hline A1 & 613 & & 362 & & 6037 & & 471 & \\
\hline $\mathrm{N} 1$, one ant, no long range & 1383 & 2.3 & 649 & 1.8 & 11,032 & 1.8 & 491 & 1.04 \\
\hline N1, two ant, no long range & 2525 & 4.1 & 983 & 2.7 & 18,051 & 3.0 & 496 & 1.05 \\
\hline $\mathrm{N} 1$, two ant + long range & 5053 & 8.2 & 1580 & 4.4 & 18,579 & 3.1 & 497.5 & 1.06 \\
\hline
\end{tabular}




\section{Increased performance through development}

AISSat-1 was pioneering maritime surveillance and ship tracking when launched in 2010 . It collected $\sim 50,000$ messages daily from the High North throughout 2010. Simulations had shown that this would be sufficient for monitoring of the High North, even with a margin for increased traffic, but that the North Sea and other hightraffic areas would not be monitored that well [14]. From 2011, the satellite has been used globally, collecting 500,000-600,000 messages daily. The ASR 100 utilizes an SDR design with two receiver channels processing the signals from an omnidirectional antenna receiving messages on AIS1 and AIS2. In 2011, the first in-orbit firmware upgrade of the receiver was made to increase the performance.

The evaluation of the receiver performance can be grouped and analyzed in three steps: the development of the receiver (electronics and the algorithms), the antenna diversity (two orthogonal monopoles), and the frequency diversity (continuous operation on all four AIS channels). The development was not necessarily made in that order; work has been carried out in parallel both on the technical side and in the standardization organizations.

The improvements can be quantified by comparing the number of messages received and ships detected with AISSat-1 and NorSat-1. The number of messages received and ships detected as well as the improvement factors due to the three developments are shown in those steps in Table 4, comparing the monthly median values of February 2018 based on data from AISSat-1 (A1) and NorSat-1 (N1) in the two areas previously discussed. The Count is the total number of messages or ships (MMSIs); the performance metric is:

Factor $=\frac{\text { The Count for NorSat- } 1 \text { in a configuration }}{\text { The Count for AISSat-1 }}$
$=\frac{\mathrm{N} 1(x \text { ant, } y \text { long-range }) \text { Count }}{\text { A1 Count }}$

where $x$ is one or two antennas, and $y$ is without/with the long-range AIS messages.

The improvements are greater in the high-traffic area than in the open ocean area. In the Mediterranean Sea, NorSat-1 shows a significant improvement for both number of messages and number of ships in all the steps. Looking at the results from the North Atlantic, an improvement in number of messages can clearly be seen, while the improvement in number of ships is marginal over a 24-h period, indicating that the daily ship detection probability by the AISSat-1 generation AIS receiver was good from the start.

\section{Summary and conclusion}

The nano- and microsatellites AISSat-1, AISSat-2, NorSat-1, and NorSat-2 provide Norwegian government users with maritime surveillance data through collection of AIS messages from ships. While safety at sea and maritime situational awareness in the High North have always been the primary focus, the satellites continuously collect messages around the world that are used by Norwegian authorities and their partners. The NORAIS- 2 receiver on ISS has served as a development environment for space-AIS receiver technology as well as services. Globally, the amounts of data have passed 4 million messages from $\sim 60,000$ ships on a daily basis counting position reports (message types $1-3,18$, and 27) only.

The improvement of the ship detection capability has been a continuous effort. Globally, NorSat -1 collect $\sim 1.5$ million position reports from $\sim 50,000$ ships per day. Comparing the global results of NorSat-1 to AISSat-2, the NorSat receives $\sim 2.8$ times more messages from $\sim 1.8$ times as many ships as the AISSat on 6 August 2017.

In high-traffic areas, the ship detection and tracking is improved even more. Using the Mediterranean Sea as example, the median values of the daily number of messages and ships in February 2018 show that the ASR x50 receivers on-board the NorSat-1 receives almost ten times as many messages as the ASR 100 receiver on-board AISSat-1, and the factor for the number of ships is larger than four. The median number of ships observed by AISSat- 1 was 362 per day, of which $27 \%$ with more than one observation per day. For NorSat-1, the numbers are 1580 ships, of which $42 \%$ had more than one observation; a significant improvement in numbers as well as the percentages. Whereas the Mediterranean is used as example of high-traffic area in this paper, results from the North Sea can be found in [15].

In the central part of the Atlantic approach to Europe, the median number of messages per day is 6037 for NorSat-1, which is $\sim 3$ times higher than for AISSat-1. The median number of ships detected daily is 497.5 for NorSat-1, which is only $3 \%$ higher than for AISSat-1. The improvement can be seen as an increased detection probability during an overpass [13]. The average daily number of observations per ship is 2.4 for AISSat- 1 and 3.7 for NorSat-1, which improve the temporal resolution and the tracking capability.

The improvements are thanks to developments in RF front-end technology, implementation of an advanced decoder, multi-antenna support, and the multi-channel architecture enabling continuous operation on all four AIS channels. The latter improvements are made possible through the addition of the long-range message that is developed to support reception of AIS messages by satellites to the AIS standard. 
Provided that more ships start using equipment supporting long-range AIS, the services will improve gradually with the current satellites through the long-range AIS capability of the ASR x50 receivers. Use of the high-gain antenna in combination with controlled satellite attitude is also expected to have impact on the performance. Further algorithm upgrades based on research and development may come on top of that.

The ASR x50 is designed to meet the requirement of microsatellite missions with a mass of $1.3 \mathrm{~kg}$, a power consumption of only $5 \mathrm{~W}$, and a 7.5 year lifetime. The AIS receiver development is continuously on going with a focus on miniaturization and low power while maintaining high flexibility and performance.

Acknowledgements The development and capabilities of the Norwegian satellite-AIS system are thanks to the investments and efforts of several organizations and individuals. The list of authors of this paper represents a few of the representatives from the Norwegian Defence Research Establishment (FFI), Kongsberg Seatex AS, the Norwegian Coastal Administration, the Norwegian Maritime Authority, Kongsberg Satellite Services AS, Statsat AS, and the Norwegian Space Agency. Many more deserve credit for their ideas, designs, and efforts; thanks to all of you!

Funding The ASR x50 receiver has been developed under ESA ARTES contract 4000110044/14/NL/NR, funded by the European Space Agency, ESA-ESTEC, Noordwijk, The Netherlands. Opinions, interpretations, recommendations, and conclusions expressed herein are those of the authors and are not necessarily endorsed by the European Space Agency.

Open Access This article is distributed under the terms of the Creative Commons Attribution 4.0 International License (http://creativeco mmons.org/licenses/by/4.0/), which permits unrestricted use, distribution, and reproduction in any medium, provided you give appropriate credit to the original author(s) and the source, provide a link to the Creative Commons license, and indicate if changes were made.

\section{References}

1. Norwegian Space Agency, NorSat-1 and NorSat-2 launched!, by Berit Ellingsen, Updated: 12.07.2017, https://www.romsenter.no/ eng/News/News/NorSat-1-and-NorSat-2-launched. Accessed 12 Apr 2018

2. European Space Agency, Norway launches advanced SatelliteAIS payloads to improve maritime shipping coverage, Published 14 July 2017 https://artes.esa.int/news/norway-launches-advan ced-satellite-ais-payloads-improve-maritime-shipping-coverage (2018). Accessed 12 Apr 2018.

3. European Space Agency, Telecom, ARTES Programme, Novel SAT-AIS Receiver Phase B2/C/D, https://artes.esa.int/projects/ novel-sat-ais-receiver-phase-b2cd. Accessed 6 Nov 2019
4. Recommendation ITU-R M.1371-5, Technical characteristics for an automatic identification system using time division multiple access in the VHF maritime mobile frequency band, International Telecommunication Union, Geneva, 2014, https://www.itu.int/ rec/R-REC-M.1371/en. Accessed 12 Apr 2018

5. Eriksen T., Bråten L.E., Skauen A.N., Haugli H.C., Løge L., Bjørnevik A., Storesund F.A.S., Alagha N.S." VDE-SAT Preliminary verification results for proposed satellite component of new maritime communications system, Proceedings of the 4S Symposium, Sorrento, Italy, 28 May 1 June 2018

6. Meld. St. 32 (2012-2013) Report to the Storting (White Paper), Between heaven and earth: Norwegian space policy for business and public benefit, approved 26 April 2013. https://www.regje ringen.no/contentassets/0307388a5ded4f50b408d3aa8c916cb1/ engb/pdfs/stm201220130032000engpdfs.pdf. Accessed 18 Nov 2019

7. Meld. St. 26 (2012-2013), Nasjonal transportplan $2014-2023$ (in Norwegian). https://www.regjeringen.no/contentassets/e6e76 84b5d54473dadeeb7c599ff68b8/no/pdfs/stm201220130026000dd dpdfs.pdf

8. Kongsberg ASR 100, AIS Space Receiver, data sheet, October 2015. https://www.kongsberg.com/maritime/contact/request-infor mation/

9. Kongsberg, ASR $\times 50$, AIS Space Receiver, data sheet, September 2016, https://www.kongsberg.com/globalassets/maritime/kmproducts/product-documents/ais-space-receiver (2019) Accessed 15 October 2019.

10. Burzigotti, P., Ginesi, A., Colavolpe, G.: Advanced receiver design for satellite-based automatic identification system signal detection. Int. J. Satell. Commun. Network 30, 52-63 (2012). https:// doi.org/10.1002/sat

11. Eriksen, T., Greidanus, H., Delaney, C.: Metrics and providerbased results for completeness and temporal resolution of satellitebased AIS services. J. Mar. Policy 93, 80-92 (2018). https://doi. org/10.1016/j.marpol.2018.03.028

12. Skauen, A.N.: Quantifying the tracking capability of space-based AIS systems, pp. 527-542. Elsevier, Amsterdam (2016). https:// doi.org/10.1016/j.asr.2015.11.028

13. Skauen, A.N.: Ship tracking results from state-of-the-art spacebased AIS receiver systems for maritime surveillance. CEAS Space J. 11, 301-316 (2019). https://doi.org/10.1007/s12567-01900245-Z

14. Olsen O., Eriksen T., Narheim B.T.: Observation Modeling Of AIS Message Reception with Satellite, FFI/RAPPORT-2007/00306, IN CONFIDENCE (2 years) i.h.t. beskyttelsesinstruksen $\$ 4$, jfr offentlighetslovens §5, 1.ledd, January 2007

15. Harr, J., Jones T., Andersen B.N., Eriksen T., Skauen A., Svenes K., Blindheim E.V., Spydevold I., Beattie A., Bradbury L.M., Cotten B., Kekez D., Mehradnia P., Zee R.E.: Frode Storesund: Microsatellites for Maritime Surveillance an update on the Norwegian Smallsat Program, IAC-18.B4.4.2x45204, 69th International Astronautical Congress (IAC), Bremen, Germany, October 2018

Publisher's Note Springer Nature remains neutral with regard to jurisdictional claims in published maps and institutional affiliations. 